\title{
Local removal of phagocytic synovial lining cells by clodronate-liposomes decreases cartilage destruction during collagen type II arthritis
}

\author{
P L E M Van Lent, A E M Holthuysen, N Van Rooijen, L B A Van De Putte, \\ W B Van Den Berg
}

\begin{abstract}
Objective-To investigate whether local removal of phagocytic synovial lining cells (SLCs) from the knee joint before onset of collagen type II arthritis has an effect on development of cartilage destruction.

Methods-Phagocytic SLCs were selectively depleted by a single injection of clodronate laden liposomes in the knee joint seven days before induction of collagen type II arthritis (CIA). Clodronate laden liposomes were given in one knee joint either alone or in combination with a short-term oral treatment of dexamethasone. Cartilage damage including proteoglycan depletion and chondrocyte death was measured in total knee joints sections stained with safranin-o or haematoxylin. Results-Local removal of phagocytic SLCs, seven days before arthritis onset, prevented cell influx for the larger part. Chondrocyte death was significantly decreased in the SLC depleted arthritic joint both at an early (6 days) and late (12 days) time point after CIA induction. However, depletion of proteoglycans from femoral and patellar cartilage layers was not prevented. If the mild acute inflammation caused by a single clodronate laden liposome injection in the left knee joint, was blocked by a short-term (on consecutive days $9,8,7,6,5$ before CIA onset) oral treatment with dexamethasone, cell influx, but also proteoglycan depletion was almost completely blocked. In the contralateral control right knee joint prominent cell influx and severe cartilage damage was observed, indicating that there was no effect of dexamethasone anymore at the onset of CIA.
\end{abstract}

Conclusions-This study shows that removal of phagocytic lining cells before CIA induction, particularly in the presence of a short-term treatment with dexamethasone, decreases cartilage destruction.

(Ann Rheum Dis 1998;57:408-413)

Destruction of cartilage is one of the main features during rheumatoid arthritis (RA) that leads to joint disability. Cartilage destruction is probably caused by substances released by both infiltrating inflammatory cells and local activated synovial cells. ${ }^{1}$ Treatments inhibiting influx of inflammatory cells and local synovial cells might be most effective in preventing cartilage destruction during RA.

In earlier studies we found that phagocytic lining cells, forming an integral part of the lining layer covering the inside of diarthrodial joints, play a crucial part in the onset of joint inflammation. ${ }^{2-4}$ It was found that selective removal of these cells from the murine knee joint by a single local injection of clodronate laden liposomes before onset of experimental arthritis, prevented the larger part of inflammation. This was found in both models expressing mild reversible inflammation like immune complex arthritis ${ }^{2}$ as well as models that develop severe irreversible inflammation like collagen type II arthritis. ${ }^{3}$ Phagocytic lining cells probably form an important source of chemokines released after activation by proinflammatory cytokines like interleukin 1 (IL1) and tumour necrosis factor (TNF). Local injection of IL1 in knee joints of normal ${ }^{2}$ or immunised $^{3}$ mice depleted of phagocytic lining cells failed to develop inflammation whereas substantial inflammation was observed in controls.

Apart from producing pro-inflammatory agents, macrophage-like lining cells also produce enzymes like collagenases, ${ }^{56}$ stromelysins, ${ }^{57}$ cathepsins, ${ }^{8}$ and proteoglycanases. ${ }^{9}$ Although these enzymes might be directly involved in cartilage degradation they are probably more important in stimulation of pro-metalloproteinases released from fibroblast-like synoviocytes. ${ }^{9}$

To investigate whether macrophage-like lining cells are involved in cartilage destruction, we selectively depleted these cells by a single local injection of clodronate laden liposomes in the knee joint of mice before arthritis induction. ${ }^{10}$ Phagocytic lining cells selectively absorb clodronate laden liposomes and subsequently die, probably of apoptosis. ${ }^{11}{ }^{12}$ As a model we used collagen type II arthritis (CIA). CIA is extensively studied to understand pathogenetic events in human arthritic joints. ${ }^{1314}$ CIA is a highly aggressive arthritis with early loss of proteoglycans and chondrocyte death leading to complete loss of articular cartilage in the later phases of arthritis. We investigated the loss of proteoglycans from the cartilage matrix and chondrocyte death by histological examination in synovial lining cell (SLC) depleted knee joints at several time points (6 and 12 days) after onset of CIA. As disease expression of CIA starts gradually 3-4 weeks after immunisation in some mice,
Accepted for publication 28 April 1998 
whereas a $100 \%$ incidence commonly takes 8-10 weeks, the onset was accelerated and synchronised by giving lipopolysaccharide (LPS) systemically at day 28 after immunisation. ${ }^{15}{ }^{16}$

Our results indicate that chondrocyte death but not proteoglycan loss is significantly blocked if the lining is eliminated seven days before CIA onset. Prevention of the local side effects in the knee joint caused by a single clodronate laden liposome injection with a short-term treatment of dexamethasone, almost complete block chondrocyte death and proteoglycan loss was seen whereas corticosteroid treatment on its own was without effect. This study shows that clodronate laden liposomes given locally to knee joints seem an effective tool to lower severe cartilage damage seen during CIA.

\section{Methods}

ANIMALS

Male DBA/1 lac J mice were obtained from Jackson (Bar Harbor, ME, USA). They were housed under semi-sterile conditions and fed a standard diet and tap water ad libitum. They were used between 10-12 weeks of age.

\section{INDUCTION OF ARTHRITIS}

Mice were immunised with bovine Collagen type II $(100 \mu \mathrm{g})$ emulsified in Freund's complete adjuvant (Difco) (Mycobacterium tuberculosis $2 \mathrm{mg} / \mathrm{ml}$ ), by a subcutaneous injection in the base of the tail. The animals were boosted at day 21 with an intraperitoneal injection of $100 \mu \mathrm{g}$ collagen type II. The onset of polyarthritis occurs around 4 to 5 weeks. The onset, incidence, and severity of arthritis is, however, highly variably within and among experiments. To synchronise the onset of arthritis in the knee joint, $40 \mu \mathrm{g}$ of bacterial LPS $^{1516}$ was given intraperitoneally at day 28 after immunisation. Using LPS, a $100 \%$ arthritis incidence is reached expressed both in hindpaws and knee joints.

LIPOSOME ENCAPSULATED DICHLOROMETHYLENE DIPHOSPHONATE

Multilamellar liposomes encapsulating the drug clodronate: dichloromethylene diphosphonate $\left(\mathrm{CL}_{2} \mathrm{MDP}\right)$ were prepared as described earlier. ${ }^{17}$ Clodronate was kindly provided by Boehringer Mannheim, Germany. Briefly 86 mg phosphatidylcholine and $8 \mathrm{mg}$ cholesterol are dissolved in chloroform in a round bottom flask. By vacuum rotary evaporation at $37^{\circ} \mathrm{C}$, a thin film formed on the wall of the flask. Clodronate $(2.5 \mathrm{~g})$, dissolved in $10 \mathrm{ml}$ phosphate buffered saline (PBS) and enclosed by gentle shaking for 10 minutes was kept at room temperature for two hours and sonicated at $20^{\circ} \mathrm{C}$ for three minutes. After an additonal two hours at room temperature, free clodronate was removed by washing three times with PBS $(20000 \mathrm{~g}, 10 \mathrm{~min})$. The natural fate of liposomes is phagocytosis by phagocytic cells.

DEPLETION OF PHAGOCYTIC LINING CELLS WITH CLODRONATE-LIPOSOMES

Six $\mu$ l of a clodronate laden liposome suspension (containing $30 \mu \mathrm{g} \mathrm{CL} \mathrm{CL}_{2} \mathrm{MDP}$ ) was injected once in the knee joints of collagen type II immunised mice, seven days before arthritis onset (at day 21 after arthritis induction). As control, PBS or PBS containing liposomes were injected. Phagocytic synovial lining cells ingest the clodronate-liposomes. During digestion the membrane of the liposome is disrupted, the clodronate sets free, and the cell dies of apoptosis. ${ }^{11}{ }^{12}$ If injected in knee joints of control C57 bl/6 mice, optimal depletion of synovial lining cells was observed seven days after injection.

In a further experiment, a single intraarticular injection of clodronate laden liposomes was combined with a short-term treatment (at day 19, 20,21, 22, and 23) of dexamethasone $(1 \mathrm{mg} / \mathrm{kg} /$ day orally)

TREATMENT PROTOCOL AND MACROSCOPIC SCORING OF ARTHITIS

From day 2 until day 6 or day 12 after start of arthritis, clinical severity was graded semiquantitavely on a scale of 0 to 2 for each hind paw, according to changes in redness and swelling: 0: no changes; 0.5 : slight; 1.0 : moderate; 1.5: marked; 2.0: maximal swelling and redness. Six and 12 days after onset of arthritis, mice were killed, knee joints were isolated, and processed for histological examination.

\section{HISTOLOGICAL EXAMINATION}

At the end of the experiments, total knee joints were dissected, fixed in $4 \%$ formaldehyde, and processed for histological examination as described earlier. ${ }^{18}$ Standardised frontal sections of total knee joints $(7 \mu \mathrm{M})$ were cut and stained with haematoxylin.

Eight semiserial sections per knee joint, each taken from a standard location of at least 10 animals per experimental group, were examined blind by two observers.

Cell influx was scored on a $0-3$ scale: 0 ; no cell influx, 1 ; minor, 2 ; moderate, 3 ; marked.

Depletion of the cartilage matrix was measured by determining the degree of loss of safranin-o staining intensity. Both patella and femur surfaces were screened. The scale was from 0 to $3+$, where $\mathrm{o}=$ no depletion, $1=$ low, $2=$ moderate, and $3=$ severe.

Chondrocyte death was determined by measuring the ratio (\%) of shrunken cells in almost empty lacunae and visible chondrocytes in patellar and femural cartilage surfaces. The shrunken cells stained positively in the TUNEL method (in vitro cell death kit) (data not shown).

\section{Results}

LOCAL DEPLETION OF SLC AND PROTEOGLYCAN DEGRADATION FROM CARTILAGE LAYERS DURING ACCELERATED CIA

Phagocytic SLCs were eliminated by a single injection of clodronate laden liposomes into the right knee joint. In the contralateral left knee joints control vehicles (PBS or PBSliposomes) were given. Previous studies showed that cell influx in the synovium was highly reduced for both neutrophils and macrophages. ${ }^{23}$ To further investigate the effect of lining depletion on proteoglycan 

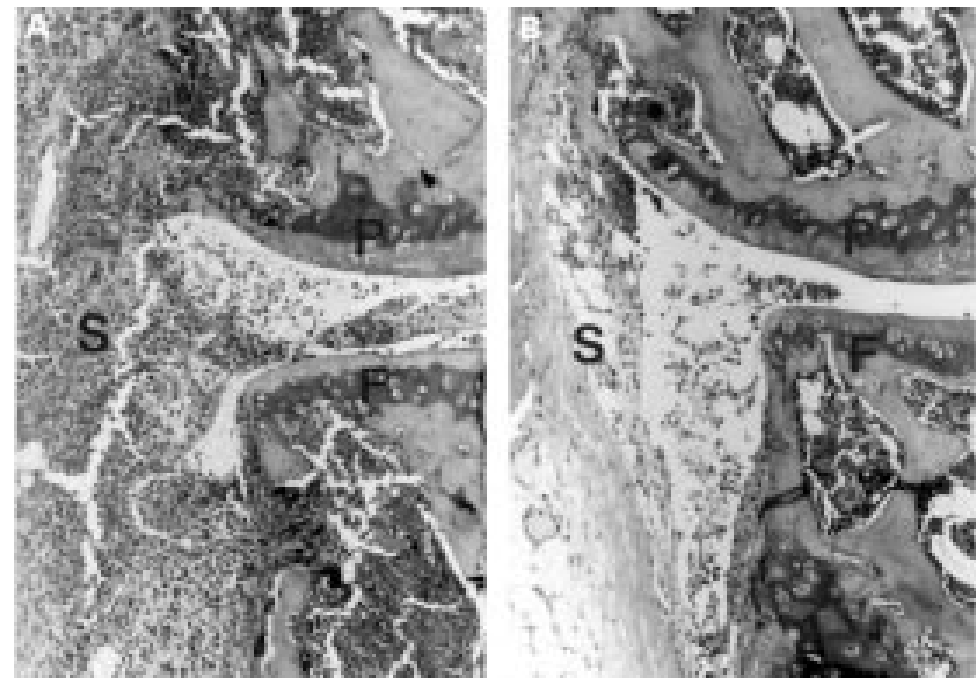

Figure 1 Total knee joint sections stained with safranin-o, six days after accelerated collagen type II arthritis. Note the significantly decreased cell influx in the arthritic knee joint in which the lining was selectively removed seven days before CIA induction $(B)$ compared with controls $(A)$. Comparable $P G$ depletion was found as seen by loss of red staining from the cartilage surface. Original magnification $\times 200$. $F=$ femur, $P=$ patella, $S=$ synovium.

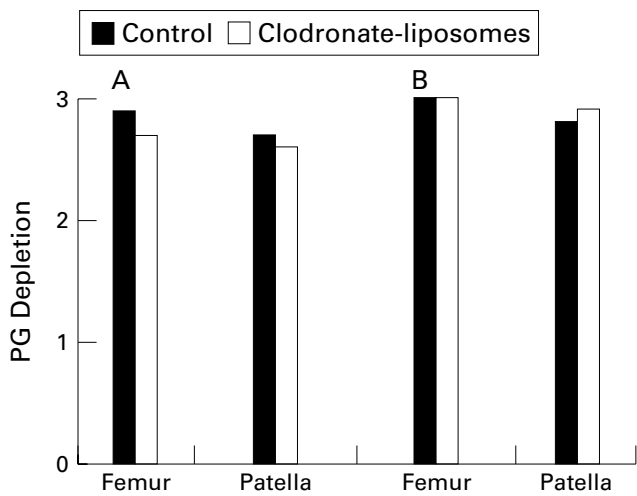

Figure 2 Depletion of proteoglycan (PG) from cartilage layer of patella and femur. PG loss was scored using an arbritrary scale from 0-3 (0: no PG loss; 1:minor; 2:moderate; 3:marked). Clodronate $\left(C L_{2} M D P\right)$ laden liposomes, PBS laden liposomes or PBS alone was injected into the knee joint before arthritis induction. Knee joints were injected seven days before arthritis onset. PG loss was scored at either day $6(A)$ or day $12(B)$ after CIA onset. Note that the PG loss in the low inflamed, clodronate treated knee joints was not significantly different from high inflamed control arthritic joints. Values represent two experiments with 10 mice per group.

degradation in cartilage layers within this model, the degree of loss of red staining in femur and patellar cartilage layer was measured in safranin-o stained total knee joint sections. Figure 1 shows that although significantly reduced cell influx was observed in the SLC depleted knee joints, proteoglycan depletion was still severe and not significantly different from controls (fig 1 (A), (B); fig 2). Comparable proteoglycan loss was measured at an early (day 6) (fig 2 (A)) and late time point (day 12) after arthritis onset (fig 2 (B)).

\section{LOCAL DEPLETION OF SLC AND CHONDROCYTE DEATH}

Chondrocyte death is a characteristic feature in classic and accelerated CIA. Total knee joint sections stained with haematoxylin showed shrinkage of cells and nuclei in the lacunae at day 6 after induction of arthritis.

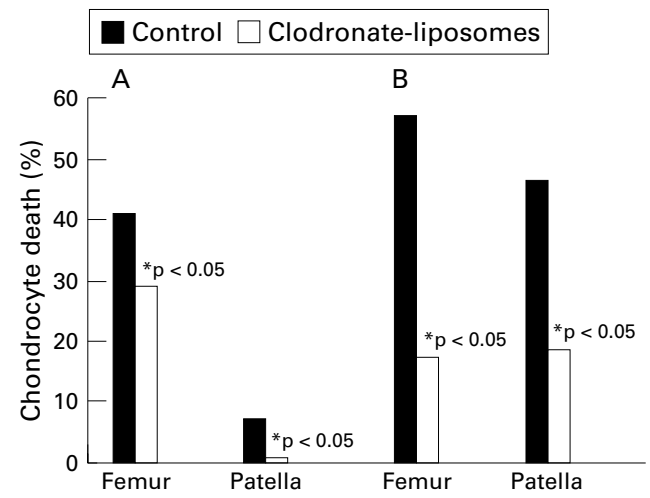

Figure 3 Chondrocyte death in patellar and femural cartilage layer of SLC depleted and control knee joints. Chondrocyte death was measured by determining the percentage chondrocyte death (empty lacunae) and total chondrocytes. Clodronate $\left(C L_{2} M D P\right)$ laden liposomes, PBS-laden liposomes or PBS alone was injected into the knee joint before arthritis induction. Knee joints were injected seven days before arthritis onset. Percentage chondrocyte death was measured at either day $6(A)$ or day 12 (B) after CIA onset. Note that chondrocyte death in the low inflamed SLC depleted arthritic knee joints is significantly lower than that from highly inflamed control arthritic joints if clodronate laden liposomes were given seven days before CIA onset. Values represent two experiments with 10 mice per group. $p<0.05$ compared with $P B S$ or PBS containing liposomes, by Mann-Whitney $U$ test.

Studying whole knee joint sections, it was found that in the first phase of control accelerated CIA (six days after onset), more chondrocyte death is observed in femural $(40 \%)$ than in patellar cartilage (only 10\%). In the later phase (day 12 after onset), chondrocyte death was significantly increased both in the femur (from 40 to $60 \%$ ) as well as in the patella (from 10 to $50 \%$ ) (fig 3 (A), fig 44 (A) (B)).

In SLC depleted joints, chondrocyte death was significantly lower both at 6 and 12 days after CIA development. At day 6, 25\% less chondrocyte death was found in the femural cartilage whereas no cell death was found in the patellar cartilage. At day 12 we found a noticeable protection against chondrocyte death both in the femur $(70 \%$ protection) and in the patella (60\% protection) (fig $3(\mathrm{~B}))$.

CLODRONATE-LIPOSOME TREATMENT COMBINED WITH DEXAMETHASONE TREATMENT

A single clodronate laden liposome injection in the knee joint of immunised mice causes synovial lining depletion but also a short-term mild inflammation, ${ }^{11}$ which may cause unwanted side effects on cartilage metabolism. To investigate whether the absence of a protective effect on cartilage proteoglycan loss upon full expression of CIA could be related to this early inflammation, mice were treated orally by dexamethasone on five alternate days (day 19, 20, 21,22 , and 23 after CIA induction) around the injection of clodronate-liposomes (day 21 after CIA induction). In the right knee joint, clodronate-liposomes were injected whereas in the left knee joint we injected PBS.

The treatment with dexamethasone in the early phase of immunisation had no effect on the macroscopic scoring of paw swelling measured from day 28 until day 34 if compared with immunised mice, which only received the 

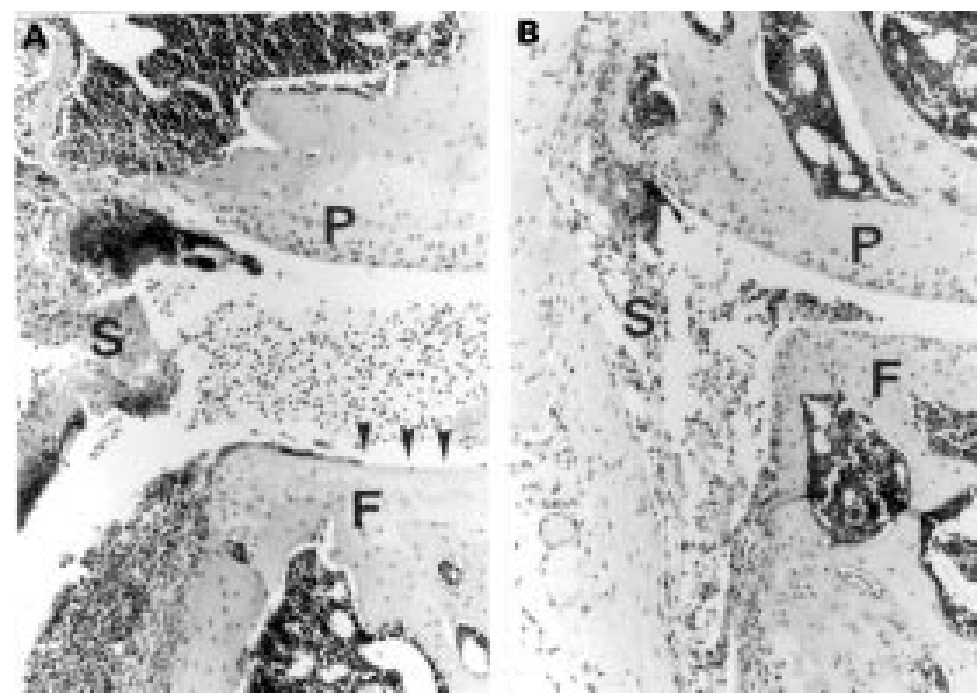

Figure 4 Total knee joint sections stained with haematoxylin and eosin, six days after accelerated collagen type II arthritis. Note the significantly decreased cell influx in the arthritic knee joint in which the lining was selectively removed seven days before CIA induction (B) compared with controls (A). Significantly less chondrocyte death is found particularly in the femur in lining depleted arthritic knee joints. Original magnification $\times$ 200. $F=$ femur, $P=$ patella, $S=$ synovium.

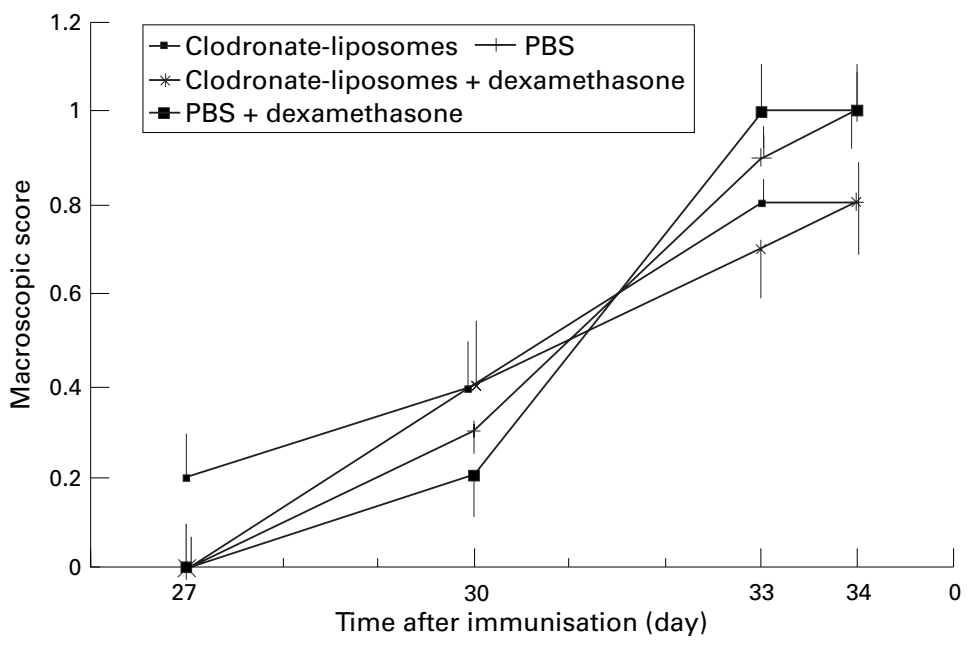

Figure 5 Clinical severity of arthritis in the hindpaws of $D B A / 1$ mice with accelerated type II collagen induced arthritis (CIA). CIA was accelerated by giving $40 \mu \mathrm{g}$ of lipopolysaccharide (LPS) on day 28. Clodronate laden liposomes were injected in the left knee joint whereas PBS was injected in the right knee joint on day 21 . One group of seven mice were treated orally with dexamethasone (1 mg/kg/day) on day 19, 20, 21, 22, 23. The control group was given the vehicle at similar time points. Clinical severity of the hind paw was scored using an arbritary scale from 0-2 per paw. Note that the clinical severity of the hind paws was not different between dexamethasone and vehicle treated animals.

vehicle at the same time points, excluding a systemic impact on the arthritis (fig 5). In the left PBS injected knee joint of dexamethasone treated mice, a full blown inflammation (fig $6(\mathrm{~A})$, fig $7(\mathrm{~A})$ ) and severe cartilage destruction in femur and patella was observed (fig 6 (B), (C), fig 7(A)). However, in the contralateral lining depleted knee joint of dexamethasone treated immunised mice, cell influx was totally prevented (fig 6(A), fig 7(B)). More interestingly only minimal proteoglycan depletion was found in both patella and femur (fig 6(B), fig 7 (B)) whereas no chondrocyte death was found in the femur (fig $6(\mathrm{C})$, fig $7(\mathrm{C})$ ). In SLC depleted knee joints of CIA mice that did not receive a systemic dexamethasone treatment, it was again confirmed that chondrocyte death was reduced but not proteoglycan depletion.

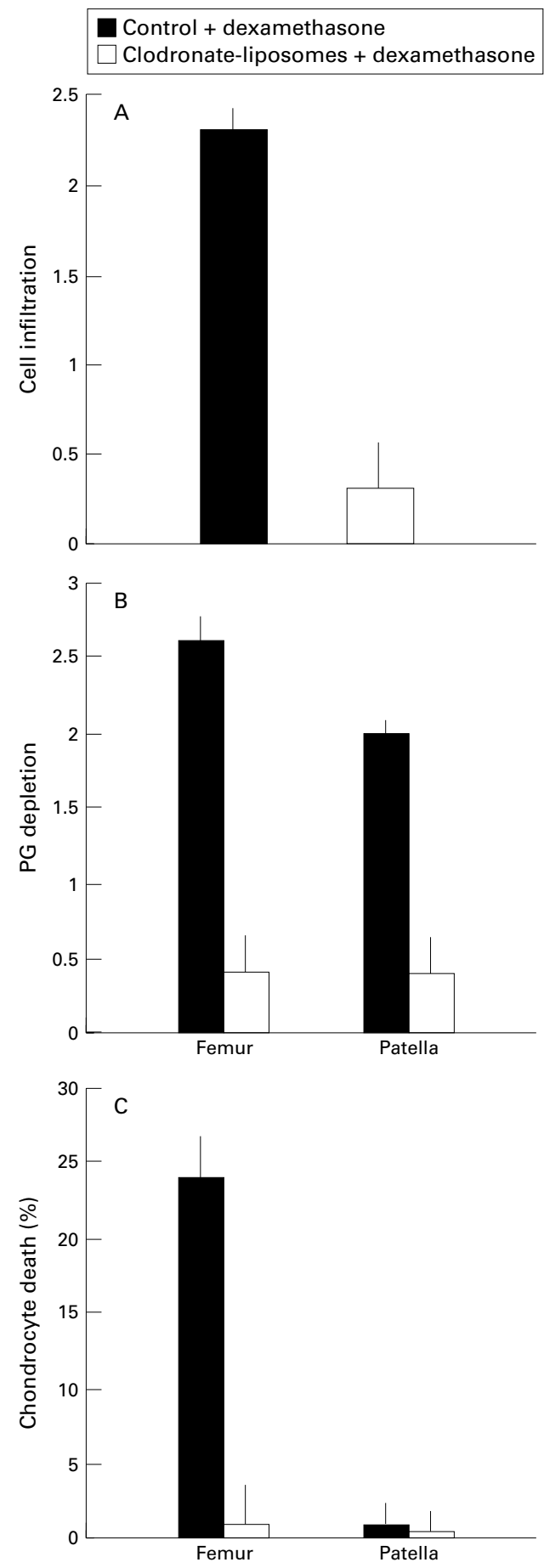

Figure 6 Cell influx, proteoglycan depletion, and chondrocyte death in knee joints of $D B A / 1$ mice with accelerated type II collagen induced arthritis (CIA). Total knee joint sections were stained with either haematoxylin and eosin (cell influx) or safranin-o (proteoglycan loss). Cell influx and proteoglycan loss was scored on a $0-3$ scale. The percentage chondrocyte death was expressed as ratio of number of empty lacunae/number of total lacunae.

Clodronate laden liposomes or PBS alone was injected in the knee joint before arthritis induction. One group of seven mice was treated orally with dexamethasone (1 $\mathrm{mg} / \mathrm{kg} /$ day) on days 19,20,21,22, 23. Note the absence of cell infiltration and chondrocyte death and the minor proteoglycan loss in SLC depleted but not control arthritic knee joints of mice given dexamethasone. The control group was given the vehicle at similar time points. Clinical severity of the hind paws was not different between dexamethasone and vehicle treated animals (data not shown). 

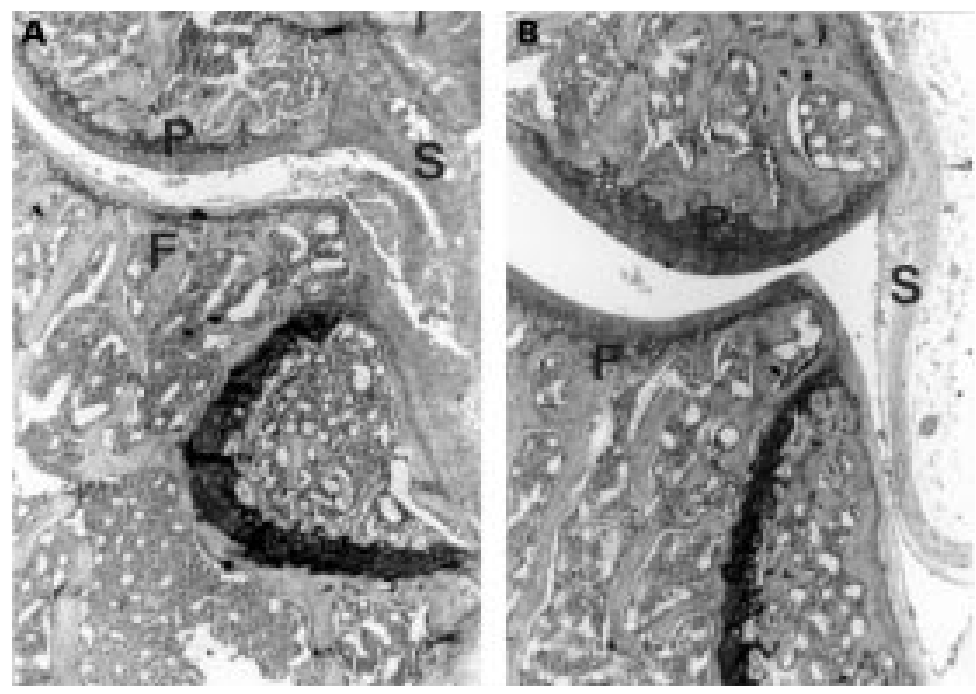

Figure 7 Total knee joint sections stained with safranin-o, six days after accelerated collagen type II arthritis that started at day 28 after immunisation. Mice were given dexamethasone (1 $\mathrm{mg} / \mathrm{kg} /$ day) orally on day 19, 20,21, 22, 23, around the intra-articular injection of clodronate laden liposomes at day 21 after immunisation. Note the significantly decreased cell influx and minor proteoglycan depletion in the arthritic knee joint in which the lining was selectively removed (B) compared with the contralateral control joint with intact lining $(A)$. Original magnification $\times 100 . F=$ femur, $P=$ patella, $S=$ synovium space.

\section{Discussion}

In a previous study we found that local lining depletion in the knee joint blocks the larger part of the expression of the inflammatory response during CIA. ${ }^{2-4}$ In this study we show that local clodronate-liposome treatment also has beneficial effects on cartilage damage, but full protection was only found if clodronateliposomes were applied in the presence of dexamethasone.

Severe cartilage destruction like inhibition of proteoglycan synthesis, degradation of proteoglycan, and death of chondrocytes is characteristic for collagen type II induced arthritis. Chondrocyte death, which was already prominent around day 6 after CIA onset, was significantly decreased in SLC depleted arthritic joints. Chondrocyte death observed within this model is probably caused by inflammatory mediators released by local synovial cells or infiltrating cells, or both. Persistent collagen type II immune complexes may activate infiltrating cells like PMN and macrophages. These cells are important producers of nitrogen and oxygen radicals, ${ }^{19}{ }^{20}$ which may lead to either apoptosis or necrosis of chondrocytes. ${ }^{21}$ As the amount of infiltrating cells is significantly reduced in SLC depleted arthritic knee joints, this may dampen the local release of radicals and thus prevent severe chondrocyte death. Activated PMN also are an important source of serine proteinases, elastase being the most prominent. ${ }^{22}$ Apart from its proteoglycan degrading capacity, ${ }^{22}{ }^{23}$ elastase also activates proforms of metalloproteinases, like stromelysin and collagenases. ${ }^{24}$ Release of these enzymes by the chondrocyte may increase erosion of the cartilage matrix and chondrocyte death.

Although expression of inflammation in SLC depleted arthritic knee joints decreased significantly, there was still a considerable loss of proteoglycans that was similar to controls, suggesting that minor inflammation can still be destructive to cartilage. A recent study in the immune complex mediated arthritis showed that IL1 ra treatment caused selective block of PMN influx but not monocyte influx, and this was insufficient to prevent proteoglycan degradation. ${ }^{25}$ Metalloproteinase ${ }^{26}$ and the not yet identified "aggrecanase" 27 produced by minor amounts of infiltrating macrophages or local cells may be responsible for the observed proteoglycan loss. Release of these enzymes is strongly activated by pro-inflammatory cytokines like $\mathrm{TNF} \alpha$ and $\mathrm{IL}^{28}{ }^{29}$ and although these cytokines are lowered in lining depleted CIA joints ${ }^{23}$ they may still have substantial effects. Complete block of inflammatory cells by anti-adhesion (anti-CR3) antibodies in accelerated CIA knee joints prevented proteoglycan loss completely (unpublished data). This again suggests that minor inflammation may still be destructive to cartilage either directly ${ }^{30}$ or indirectly by activating synovial fibroblasts ${ }^{30}$ resulting in full blown proteoglycan depletion. In contrast with this is our previous finding in non- $T$ cell mediated immune complex arthritis in which lining depletion before arthritis induction did not only prevent the larger part of cell influx but also around $50 \%$ of the proteoglycan depletion. ${ }^{2}$ This suggests that joint structures of mice that are previously immunised by complete Freund's adjuvant like in the CIA might be more vulnerable for a small arthritic insult than joints from normal, non-immunised mice. Moreover, strain differences might also be important. Immune complex arthritis elicited in knee joints of DBA/ 1 mice is much more destructive to cartilage compared with arthritis raised in knee joints of $\mathrm{C} 57 \mathrm{Bl} / 6$ mice (unpublished data). We further investigated whether we could improve the benificial effects of clodronate-liposome treatment on cartilage damage. In earlier studies, we found that a single injection of clodronate-laden liposomes in the knee joint causes a mild, short-lasting (1-2 days) inflammation and some cartilage damage. ${ }^{10}$ To block only these short-lasting side effects of clodronate laden liposomes and not the onset of arthritis, dexamethasone was given shortly before and for a few days after liposome treatment. Dexamethasone has well characterised anti-inflammatory actions. ${ }^{31-33}$ As the biological half life of dexamethasone action is between 36-72 hours, its beneficial effects wane shortly after the last injection and we found that it did not interfere with the onset of CIA five days later. Apart from the fact that the antiinflammatory effects of dexamethasone may have subsided for the larger part at the time of onset, the use of LPS to synchronise the onset may overcome the rest inhibiting effect of dexamethasone, which leads to development of arthritis similar to the control group. Interestingly, in mice treated with dexamethasone, only minor proteoglycan loss was observed in SLC depleted knee joints whereas in the contralateral control joint, a full blown arthritis developed, showing severe proteoglycan loss. One of the dexamethasone effects is its beneficial effect on prevention of proteoglycan loss. ${ }^{29}$ 
Proteoglycan content in the cartilage matrix is the net effect of proteoglycan degradation and proteoglycan synthesis. Dexamethasone ameliorates both increased proteoglycan degradation and inhibition of proteoglycan synthesis seen during arthritis. Apart from preventing cartilage damage, dexamethasone also efficiently downregulates inflammation. ${ }^{32}$ As knee joints of collagen type II immunised mice are highly vulnerable, local injection of clodronate-liposomes may apart from depleting SLCs also induce minor synovial inflammation thus priming the joint for a second arthritic insult. The short-term treatment of dexamethasone may inhibit this minor inflammation thus preventing a flare effect by the LPS treatment at day 28.

This study shows that local treatment of clodronate-liposomes in the presence of low concentrations of dexamethasone before arthritis induction ameliorates cartilage destruction during collagen type II induced arthritis. As local treatment of clodronate-liposomes decreases both expression of inflammation and cartilage damage it might be an effective treatment for joints suffering from RA.

This study was financally supported by the Maartens Clinic, Nijmegen, the Netherlands.

1 Harris ED. Pathogenesis of rheumatoid arthritis: a disorder associated with disfunctional immunoregulation. In:Gallin JI, Goldstein IM, Snyderman R, eds. Inflammation: basic $\mathrm{JI}$, Goldstein IM, Snyderman R, eds. Inflammation: basic
principles and clinical correlates. New York: Raven Press, principles and

2 Van Lent PLEM, Van den Hoek AEM, Van den Bersselaar LAM, Sparijaards MFR, van Rooijen N, Dijstra CD, et al. In vivo role of phagocytic lining cells in onset of experimental arthritis. Am J Pathol 1993;143:1226-37.

3 Van Lent PLEM, Holthuysen AEM, van den Bersselaar L, van Rooijen N, Joosten LAB, van de Loo FAJ, et al. Phagocytic lining cells determine local expression of inflammation in type II collagen-induced arthritis. Arthritis Rheum 1996;39:1545-55.

4 Van Lent PLEM, van de Hoek A, van den Bersselaar L, Dijkstra CD, van Rooijen N, van den Berg WB. Role of synovial macrophages in experimental arthritis. Res Immunol 1992;143:229-34

5 Okada Y,Takeuchi N,Gonoji Y, Nakanishi I, Nagase H, Hayakawa T. Simultaneous production of collagenase, matrix metalloproteinase 3(stromelysin) and tissue inhibitor of metalloproteinases by rheumatoid synovial lining cells. Matrix Suppl 1992;1:398-9.

6 Maeda S, Sawai T, Uzuki M, Takahashi Y, Omoto H, Seki M, et al. Determination of interstitial collagenase(MMP-1) in patients with rheumatoid arthritis. Ann Rheum Dis in patients with

7 Nawrocki B, Polette M, Clavel C, Morrone A, Eschard JP, Etienne JC, et al. Expression of stromelysin 3 and tissue inhibitors of matrix metalloproteinases, TIMP-1 and TIMP-2, in rheumatoid arthritis. Pathol Res Pract 1994;190:690-6.

8 Keyszer GM, Heer AH, Kriegsmann J, Geiler T, Traband A, Keysser M, et al. Comparative analysis of cathepsin $\mathrm{L}$,cathepsin $\mathrm{D}$ and collagenase messenger RNA expression in synovial tissues of patients with rheumatoid arthritis and osteoarthritis, by in situ hybridization. Arthritis Rheum 1995;38:976-84.

9 Zvaifler NJ. Macrophages and the synovial lining. Scand J Rheumatol 1995;24 (suppl 101):67-75.

10 Van Lent PLEM, Van den Bersselaar L, Van den Hoek AEM, van de Ende M, Dijkstra CD, van Rooijen N, et al. Reversible depletion of synovial lining cells after intraarticular treatment with liposome encapsulated dichloromethylene diphosphonate. Rheumatol Int 1993;13:2130 .
11 Van Rooijen N, Sanders A,van den Berg TK. Apoptosis of macrophages induced by liposome-mediated intracellular delivery of clodronate and propadimine. J Immunol Methods 1996;193:93-9.

12 Smith-Weber CB, Rittig M, Buchner E, Hauser I, Schmidt I, Palombo-Kinne E, et al. Apoptotic cell death in activated monocytes following incorporation of clodronateliposomes. J Leukoc Biol 1996;60:230-44.

13 Trentham DE, Townes AS, Kang AH. Autoimmunity to colagen type II collagen: an experimental model of arthritis. J Exp Med 1977;146:857-68.

14 Stuart JM, Cremer MA, Kang AH, Townes AS. Collageninduced arthritis in rats. Evaluation of early immunological events. Arthritis Rheum 1979;22:1344-51.

15 Caccese RG, Zimmerman JL, Carlson RP. Bacterial lipopolysaccharide potentiates type II collagen-induced arthritis in mice. Mediators of Inflammation 1992;1: 273-9.

16 Joosten LAB, Helsen MMA, van den Berg WB. Accelerated onset of collagen-induced arthritis by remote inflammation. Clin Exp Immunol 1994;97:204-11.

17 Van Rooijen N, Sanders A. Liposome mediated depletion of macrophages: mechanism of action, preparation of liposomes and applications. J Immunol Methods 1994;174:8393.

18 Van den Berg WB, Van de Putte LBA, Zwarts WA, Joosten LAB. Electrical charge of the antigen determines intrarticular antigen handling and chronicity of arthritis in mice. J Clin Invest 1984;74:1850-9.

19 Hibbs JB, Taintor RR, Vavrin Z, Rachlin EM. Nitric oxide: a cytotoxic activated macrophage effector molecule. Biochem Biophys Res Commun 1988;157:87-94.

20 Wright CD, Mulsch A, Busse R, Oswald H. Generation of nitric oxide by human neutrophils. Biochem Biophys Res Commun 1989;160:813-19.

21 Blanco FJ, Ochs RL, Schwarz H, Lotz M. Chondrocyte apoptosis induced by nitric oxide. Am J Pathol 1995;146: 75-85.

22 Janoff A,Feinstein G, Malemud CJ,Elias JM. Degradation of cartilage proteoglycan by human leucocyte granule neutral proteases model of injury.I Penetration of enzyme into rabbit articular cartilage and release of ${ }^{35} \mathrm{SO}$ labelled material from the tissue. J Clin Invest 1976;57:615-24.

23 Schalkwijk J, Van den Berg WB, Van de Putte LBA, Joosten LA. Elastase secreted by activated polymorphonuclear leucocytes causes chondrocyte damage and matrix degradation in intact articular cartilage:escape from in activation by alpha-1-proteinase inhibitor. Br J Exp Pathol 1987;68: $81-8$

24 Kleiner DE Jr,Stetler-Stevenson G. Structural biochemistry and activation

Dilom A, Holthuysen AEM, Jacobs CWM, van de Putte LBA, van den Berg WB. Monocytes/macrophages rather than PMN are involved in early cartilage degradation in cationic immune complex arthritis in mice. J Leukoc Biol 1997;61:267-78.

26 Henderson B, Blake S. Connective tissue destruction in rheumatoid arthritis: therapeutic potential of selective metalloproteinase inhibitors. Immunopharmacology of joints and connective tissue. London: Academic Press, 1994:199-223.

27 Lohmander S, Neame PJ, Sandy JD. The structure of aggrecan fragments in human synovial fluid: Evidence that aggrecanase mediates cartilage degradation in inflammatory joint disease, joint injury and osteoarthritis. Arthritis Rheum 1993;36:1214-22.

28 MacNaul KL, Chartrain N, Lark M, Tocli MJ, Hutchinson NI. Discoordinate expression of stromelysin, collagenase and TIMP in human rheumatoid synovial fibroblasts: synergistic effects of IL-1 and TNF on stromelysine synergistic effects of IL-1 and TNF on

29 Pelletier JP, DiBattista JA, Roughly P, McCollum R, MartelPelletier J. Cytokines and inflammation in cartilage degra-
dation. Rheum Dis Clin North Am 1993;19:545-68.

30 Janusz MJ, Hare M. Cartilage degradation by cocultures of transformed macrophage and fibroblast cell lines: a model of metalloproteinase-mediated connective tissue degradation. J Immunol 1993;150:1922-31

31 Frisch SM, Ruley HE. Transcription from the stromelysin promoter is induced by interleukin- 1 and repressed by dexamethasone. J Biol Chem 1987;262:16300-4.

32 Hubbard JR, Mattmueller DR, Steinberg JJ, Poppas DP, Sledge CB. Effect of steroid hormones on endotoxinmediated cartilage. Mol Cell Biochem 1988;79:31-7.

33 Jafari HS, Saez-Llorens X, Paris M, Rinderknecht S, Friedland I, Ehrett S, et al. Dexamethasone attenuation of cytokine-mediated articular cartilage degradation in experimental lapine haemophilus arthritis. J Infect Dis 1993;
168:1186-93. 